Elsevier Editorial System(tm) for Sensors \& Actuators: B. Chemical

Manuscript Draft

Manuscript Number: SNB-D-07-00673

Title: Use of a low refractive index prism in surface plasmon resonance biosensing

Article Type: Research Paper

Keywords: surface plasmon resonance; biosensor; refractive index; glass prism; effective 3-layer model.

Corresponding Author: Dr. Jun Kondoh,

Corresponding Author's Institution: Shizuoka University

First Author: Gaurav Gupta, Master of Science (Physics)

Order of Authors: Gaurav Gupta, Master of Science (Physics); Mitsunori Sugimoto; Yoshikazu Matsui; Jun Kondoh

Abstract: A way to improve the angular sensitivity of surface plasmon resonance (SPR) biosensor by tuning the resonance position to a higher incident angle region using a lower refractive index glass prism is described in this paper. A novel effective 3-layer (E3L) model is described to transform a multiple layer biosensor configuration in context of SPR condition. The E3L model supports the use of a low refractive index prism for biosensing. The performance of the sensor in immunosensing is checked for two glass prisms of different refractive index materials. The experimental results showed an enhancement in the amount of resonance angle shift of the immunosensor for the lower refractive index glass prism. 


\section{Use of a low refractive index prism in surface plasmon} resonance biosensing

Gaurav Gupta $^{\text {a }}$, Mitsunori Sugimoto ${ }^{\text {b }}$, Yoshikazu Matsui ${ }^{\text {c }}$, Jun Kondoh ${ }^{\text {a, c, d* }}$

${ }^{a}$ Graduate School of Electronic Science and Technology, Shizuoka University, 3-5-1

Johoku, Hamamatsu, Shizuoka 432-8561, Japan

${ }^{b}$ Research Institute of Electronics, Shizuoka University, 3-5-1 Johoku, Hamamatsu, Shizuoka 432-8561, Japan

${ }^{c}$ Faculty of Engineering, Shizuoka University, 3-5-1 Johoku, Hamamatsu, Shizuoka 432-8561, Japan

${ }^{d}$ Graduate School of Science and Technology, Shizuoka University, 3-5-1 Johoku, Hamamatsu, Shizuoka 432-8561, Japan

*Corresponding author. Tel/Fax: $+81-53-478-1221$

E-mail: j-kondoh@sys.eng.shizuoka.ac.jp 


\begin{abstract}
A way to improve the angular sensitivity of surface plasmon resonance (SPR) biosensor by tuning the resonance position to a higher incident angle region using a lower refractive index glass prism is described in this paper. A novel effective 3-layer (E3L) model is described to transform a multiple layer biosensor configuration in context of SPR condition. The E3L model supports the use of a low refractive index prism for biosensing. The performance of the sensor in immunosensing is checked for two glass prisms of different refractive index materials. The experimental results showed an enhancement in the amount of resonance angle shift of the immunosensor for the lower refractive index glass prism.
\end{abstract}


Keywords: surface plasmon resonance, biosensor, refractive index, glass prism, effective 3-layer model.

\section{Introduction}

For the past two decades, the phenomenon of surface plasmon resonance (SPR) has been chiefly exploited in biosensing for studying various types of biochemical interactions [1], [2], [3]. An SPR based biosensor is still gaining popularity due to its easy, efficient and label-free detection. It is important to improve the sensor's sensitivity by optimizing its design parameters. The main transducing parameters are incident wavelength, metal film properties (material and film thickness) and prism material. Previous work by Homola et al. [4] showed that a smaller wavelength and gold as the metal film would result in a higher angular sensitivity. In a recent investigation on a prism-type SPR sensor by Gupta et al. [5], it was concluded that the choice of glass prism material was also an important consideration for optimizing the angular sensitivity of the sensor. Both numerical and experimental results in [5] showed that on reducing the refractive index of prism the SPR position could be tuned to a higher angle region and the angular sensitivity could be increased. In most of the reported work on SPR biosensor, either a high refractive index glass prism is used [6] due to the high refractive index of biochemicals or the SPR position lies in a low angle region, near $40^{\circ}-50^{\circ}$ [7], [8]. This compromises the angular sensitivity of the sensor.

In this paper, it is proposed to use a low refractive index prism in an SPR biosensor to tune the resonance region to a possible higher angle region and increase the 
angular sensitivity. For this a novel effective 3-layer (E3L) model is proposed that supports the use of a low refractive index prism in SPR biosensor even for a high refractive index biomolecular layer. Further, the experiments on SPR immunosensor to detect antibody-antigen binding are carried out with two prisms, one with a high refractive index of 1.597 and other with a low refractive index of 1.456 . The results are compared chiefly for the improvement in the angular sensitivity of the sensor.

\section{Effective 3-layer (E3L) model}

An SPR biosensor configuration is described as a multi-layer model consisting of a glass prism, a thin metal film, and various thin biomolecular layers in buffer environment (Fig. 1(a)). The presence of different layers makes the configuration complex. The refractive index of biomolecular layer is in the range $1.45-1.55$ [9] and that of buffer solution is in the range $1.33-1.34$. Due to the high refractive index of bio-layers, a high refractive index prism is used. This is to satisfy the SPR condition, as explained later.

The principle of an SPR sensor is that the resonance condition is sensitive to the refractive index of the dielectric media in the vicinity of the metal film [10]. The surface plasma wave (SPW) generated at the metal-dielectric interface at resonance is evanescent inside the dielectric medium. Since this SPW is active within its penetration depth and the penetration depth $(\sim 200-300 \mathrm{~nm})$ being much larger than the thickness of the biomolecular layers $(\sim 30-40 \mathrm{~nm})$, the resonance condition must be dependent not only on the refractive index of the biomolecular layer but also on the surrounding buffer solution's refractive index. Thus there must be an effective refractive index value lying between the indices of bio-layer and buffer solution that contributes to the resonance 
condition. The $\mathrm{E} 3 \mathrm{~L}$ model is proposed to estimate that effective refractive index between the bio-layer and the buffer solution in context of SPR. For this, the multiple media SPR biosensor configuration is converted to a three media SPR configuration. All the dielectric layers are hypothetically replaced by an effective single dielectric layer (Fig. 1(b)). The concept of conversion is to find a single dielectric layer with such a refractive index value whose contribution to the SPR condition is identical to the combined effect of all the bio-layers and the buffer solution. Below an example is described to transform a 4-layer configuration to an E3L configuration keeping the same SPR characteristics.

Consider a 4-layer biosensor configuration, as shown in Fig. 2(a), consisting of a glass prism of refractive index $\left(n_{\mathrm{p}}\right) 1.70$, a $50 \mathrm{~nm}\left(d_{\mathrm{m}}\right)$ thick gold film with dielectric constant $\left(\varepsilon_{\mathrm{m}}\right)-14.77+\mathrm{i} 1.18$ (at $\lambda \sim 670 \mathrm{~nm}$ ) [11] and a thin bio-layer of refractive index $\left(n_{\text {bio }}\right) 1.45$ with variable thickness $\left(d_{\text {bio }}\right)$ surrounded by a buffer solution of refractive index ( $\left.n_{\text {buf }}\right) 1.335$. Assuming the value of $d_{\text {bio }}$ to be $10 \mathrm{~nm}$ (let's say) the angular reflection spectrum of a transverse-magnetic (TM) polarized wave incident at prism-gold interface was numerically generated for the 4-layer configuration of Fig. 2(a). The resonance angle of the spectrum was noted as $\theta_{\mathrm{sp}}{ }^{\prime}$. The spectrum was generated using Fresnel reflection coefficients [12]. Then a 3-layer configuration as shown in Fig. 2(b) was considered with a single dielectric medium of unknown effective refractive index $\left(n_{\text {eff }}\right)$. The angular reflection spectrum for 3-layer configuration of Fig. 2(b) was numerically generated with starting value of $n_{\text {eff }}$ as 1.335 (n $\left.n_{\text {buf }}\right)$. The value $n_{\text {eff }}$ was increased in steps of 0.0001 till the resonance angle of the spectrum fitted exactly with the previous value of $\theta_{\mathrm{sp}}{ }^{\prime}$ obtained for 4-layer configuration. The corresponding value of $n_{\text {eff }}$ was noted as the refractive index of the single dielectric layer. Figure 3 shows the 
numerical SPR spectra for 4-layer and E3L configuration with SPR at the same angle $\left(\theta_{\mathrm{sp}}{ }^{\prime}\right)$.

Conceptually, this means that the SPW gets excited at the incident angle value of $\theta_{\mathrm{sp}}$ ' for a single dielectric layer with the refractive index equal to $n_{\mathrm{eff}}$, and also for a $10 \mathrm{~nm}$ thick bio-layer in the buffer solution. Performing the calculation for $d_{\text {bio }}=10 \mathrm{~nm}$ and other data of Fig. 2 at $\lambda=670 \mathrm{~nm}$, the value of $n_{\text {eff }}$ was found to be 1.3448 and for both the configurations the resonance was observed at $\theta_{\mathrm{sp}}{ }^{\prime}=57.72^{\circ}$. In this way a 4-layer SPR configuration was transformed to an E3L configuration in context of SPR.

Further, the value of $d_{\text {bio }}$ was increased in small steps and the corresponding value of $n_{\text {eff }}$ was obtained in each case. Figure 4 plots the values of $n_{\text {eff }}$ as a function of $d_{\text {bio. }}$. For $d_{\text {bio }}=0$ i.e. no bio-layer, $n_{\text {eff }}$ took the value of 1.335 equal to $n_{\text {buf. }}$ The value of $n_{\text {eff }}$ increased with $d_{\text {bio. }}$. The value of $n_{\text {eff }}$ was closer to either $n_{\text {bio }}$ or $n_{\text {buf }}$ depending on their dominance. The thicker the bio-layer, the dominating it was. For $d_{\text {bio }}$ value beyond 300 $\mathrm{nm}$ the value of $n_{\text {eff }}$ asymptotically approached to 1.45 , the value of $n_{\text {bio. }}$. Beyond this thickness, which was more than the penetration depth of SPW, the SPW properties and the resonance position were not influenced by the changes in the dielectric media above the metal layer. Thus $n_{\text {eff }}$ was constant.

To check the validity of the conversion of 4-layer SPR configurations to E3L configuration the time-averaged square field distribution of EM wave was checked at the resonance angle. The time-averaged square field is proportional to the energy of EM wave [13]. Figures 5(a) and 5(b) plot the simulated time-averaged square field distribution across the media for 4-layer and E3L configurations, respectively, at the same incident angle and wavelength. With $d_{\text {bio }}=50 \mathrm{~nm}$, the value of $n_{\text {eff }}$ came out be 
1.3807 and $\theta_{\mathrm{sp}}$ was $60.56^{\circ}$ at $\lambda=670 \mathrm{~nm}$. The time-averaged square fields at the metal boundary were identical for both the configurations, only the distribution in the dielectric media differed. This confirmed that the characteristics of SPW at the metal-dielectric interface of 4-layer configuration were same as that of the converted E3L configuration.

Note that for the TM-polarized field, $\left\langle E_{\| z}{ }^{2}\right\rangle$ was discontinuous at the interfaces because of the field discontinuity condition but $\left\langle E_{\| x}{ }^{2}\right\rangle$ and $\left\langle H_{\perp y}{ }^{2}\right\rangle$ were continuous. This concept can be similarly extended to convert the system with more number of dielectric layers to an E3L configuration.

\section{Use of low refractive index prism in SPR biosensor}

The SPR equation is written as [10]

$$
n_{p} \sin \theta_{s p}=\operatorname{Re}\left(\sqrt{\frac{\varepsilon_{m} n_{d}^{2}}{\varepsilon_{m}+n_{d}^{2}}}\right),
$$

where $n_{\mathrm{p}}$ denotes the refractive index of prism, $n_{\mathrm{d}}$ denotes the refractive index of dielectric medium, $\varepsilon_{\mathrm{m}}$ is the dielectric constant of metal and $\theta_{\mathrm{sp}}$ is the SPR angle. 'Re' denotes the real part of the term in bracket. In order to satisfy Eq.(1) the following condition of

$$
n_{p}>\operatorname{Re}\left(\sqrt{\frac{\varepsilon_{m} n_{d}{ }^{2}}{\varepsilon_{m}+n_{d}{ }^{2}}}\right),
$$

must be satisfied to get

$$
\sin \theta_{s p}<1,
$$


such that $\theta_{s p}<90^{\circ}$.

This condition causes the selection of prism with $n_{\mathrm{p}}$ more than $n_{\mathrm{d}}$. The higher the value of $n_{\mathrm{p}}$, the lower will be the $\theta_{\mathrm{sp}}$. Now, because the value of $n_{\text {bio }}\left(=n_{\mathrm{d}}\right)$ is $\sim 1.45$ or more, above condition says that $n_{\mathrm{p}}$ value should be more than 1.6. Thus in practice, a glass prism with $n_{\mathrm{p}}$ values in the range $1.7-1.8$ is selected. Working with a high $n_{\mathrm{p}}$ value, the $\theta_{\mathrm{sp}}$ value lies in the low angle region of $40^{\circ}-50^{\circ}$.

In previous section, it was shown that a multi-layer biosensor configuration can be converted to an E3L configuration having a single dielectric layer (over the metal film) with an effective refractive index $\left(n_{\text {eff }}\right)$ whose value depends on biomolecular layers and surrounding buffer solution. So, Eq.(1) is rewritten for E3L model as

$$
n_{p} \sin \theta_{s p}=\operatorname{Re}\left(\sqrt{\frac{\varepsilon_{m} n_{e f f}^{2}}{\varepsilon_{m}+n_{e f f}^{2}}}\right)
$$

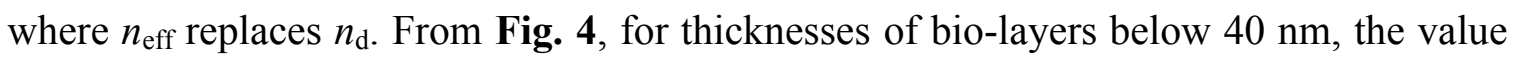
of $n_{\text {eff }}$ lies in the range $\sim 1.335-1.36$. Thus, Eq.(2) can be satisfied for a prism with $n_{\mathrm{p}}$ value near 1.46. These calculations show that even if the value of $n_{\text {bio }}$ is high, near 1.45 , a prism with $n_{\mathrm{p}}$ value 1.46 can be used for biosensing. The value of $n_{\mathrm{p}}$ to be used must be

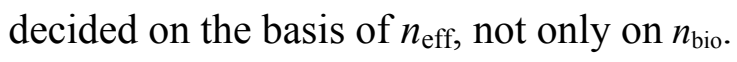

\section{Angular sensitivity}

The analytical equation of angular sensitivity $\left(S_{\theta}\right)$ can be obtained by differentiating Eq.(2) for $\theta_{\mathrm{sp}}$ with respect to $n_{\mathrm{eff}}$ and is given by 


$$
S_{\theta}=\frac{d \theta_{s p}}{d n_{\text {eff }}}=\frac{\operatorname{Re}\left(\frac{\varepsilon_{m}}{\varepsilon_{m}+n_{\text {eff }}{ }^{2}}\right)^{3 / 2}}{\sqrt{n_{p}{ }^{2}-\left\{\operatorname{Re} \sqrt{\frac{\varepsilon_{m} n_{\text {eff }}^{2}}{\varepsilon_{m}+n_{\text {eff }}^{2}}}\right\}^{2}}}
$$

For $20 \mathrm{~nm}$ thick bio-layer, $n_{\mathrm{eff}}$ is 1.3543 . Suppose an $n_{\mathrm{p}}$ value of 1.70 is selected then numerically it will result in $\theta_{\mathrm{sp}}$ value of $58.11^{\circ}$ and $S_{\theta}$ value of $78.14 \%$ RIU. But if a low value of 1.46 for $n_{\mathrm{p}}$ is selected, $\theta_{\mathrm{sp}}$ will become $80.91^{\circ}$, which is in the measurable high angle region and $S_{\theta}$ will be $351.41^{\circ} / \mathrm{RIU}$. These calculations prove that $S_{\theta}$ will increase 4 times for the lower $n_{\mathrm{p}}$ value.

The key is to reduce the $n_{\mathrm{p}}$ value of the glass prism till the resonance position lies in the measurable higher incident angle region and increase the sensitivity of the sensor system. As a result, the limit of detection (smallest detectable change in the refractive index of sample) will improve and much lower concentrations of biochemicals can be detected.

\section{Experimental}

\subsection{Sensor System}

Figure 6 illustrates a prism-based SPR sensor system. The central part comprised a glass prism over which a freshly prepared sensor chip was optically linked using an index matching liquid. The sensor chip was prepared using a glass plate of the same material as the prism. On top of the sensor chip, a silicon rubber pool was attached to hold the sample liquid. A monochromatic light from a laser diode (LD) at wavelength, $\lambda \sim 670 \mathrm{~nm}$ was made incident on a beam splitter (BS). One-half of the signal was passed through a 
polarizer $(\mathrm{P})$ to make it p-polarized and other half was made incident on photodiodel (PD1). The signal from PD1 was fed to a current-to-voltage converter (I-V converter) and used as a reference signal (Ref) to normalize the sensor signal. The light from $\mathrm{P}$ was made incident on the glass prism which refracted through to reach the prism-metal interface. The reflected light from the prism-metal interface refracted out from the prism and fell on photodiode2 (PD2). The signal from PD2 was I-V converted and fed to an analog-to-digital converter (ADC) interfaced to a computer. The normalized reflected intensity was recorded as a function of incident angle $(\theta)$ using a computer.

\subsection{Sensor chip fabrication}

A sensor chip was fabricated by depositing a thin gold film on a glass plates. The glass plates were cleansed thoroughly before the metal film deposition. At first the plates were washed with acetone. Then they were ultrasonically cleaned in acetone for 10 minutes followed by ultrasonic cleaning in 2-propanol for 5 minutes. After this they were dried using an air-pump and arranged on a substrate holder in a vacuum deposition chamber. A thin chromium film of thickness close to 2-3 nm was deposited followed by the deposition of gold film of $50 \mathrm{~nm}$ thickness. The deposition was carried out at $3 \times 10^{-6}$ Torr. Chromium was used for strong bonding of gold to the glass plate. After the film preparation, the chip was annealed on a hot plate, covered with a glass lid, for 1 hour at $300{ }^{\circ} \mathrm{C}$ (hot plate temperature).

\subsection{Chemicals and their preparation}

Dithiobissuccinimide propoinate (DSP), dimethylsulfoxide (DMSO), ethanolamine 
hydrochloride, Protein A, bovine serum albumin (BSA), rabbit anti-BSA, casein blocking buffer, phosphate buffer saline (PBS), Tween20 were purchased from Sigma (Switzerland). $4 \mathrm{mg} / \mathrm{ml}$ DSP solution was prepared in DMSO solution. 1M ethanolamine hydrochloride and $0.01 \mathrm{M}$ PBS solutions were prepared in distilled water. $0.01 \mathrm{M}$ PBS with $0.05 \%$ Tween 20 solution was prepared separately as a washing buffer (PBST). 1.0 $\mathrm{mg} / \mathrm{ml}$ Protein A, $0.25 \mathrm{mg} / \mathrm{ml}$ anti-BSA and $0.25 \mathrm{mg} / \mathrm{ml} \mathrm{BSA}$ solutions were prepared in 0.01M PBS buffer solution. Casein solution was not diluted and used at the concentration as purchased. Protein A, BSA, anti-BSA, PBS buffer, PBST and casein were kept at $4^{\circ} \mathrm{C}$ when not used.

\subsection{Immunosensor experiment}

In the described immunosensor, antibody molecules were immobilized on the sensor surface and the response of the sensor for antigen binding was observed. The antibody used was rabbit anti-BSA and the antigen was BSA. A uniform and stable immobilization of antibody is important for a sensitive detection of antigen. Recently, Schmid et al. [6] described a reliable site-directed immobilization technique for the immobilization of antibody molecules on a thiolated gold film via protein A. A similar protocol was followed in this paper and the anti-BSA - BSA binding was carried out.

A silicon pool was attached on a freshly prepared sensor chip. $4 \mathrm{mg} / \mathrm{ml}$ DSP solution was added to the pool and kept for 24 hours at room temperature. The chip was then washed with DMSO solution to remove the unbound thiol molecules. Then $1 \mathrm{mg} / \mathrm{ml}$ Protein A solution was added to the pool and kept in the refrigerator at $4{ }^{\circ} \mathrm{C}$ for 4 hours. The chip was taken out and rinsed with PBST. After this $1 \mathrm{M}$ ethanolamine hydrochloride was 
added and kept for 1 hour for blocking the residual (unbound) sites. The blocking procedure minimizes the non-specific binding. Then the chip was thoroughly rinsed with distilled water, the PBS buffer was added to the pool and the chip was attached to the prism in the sensor system.

At first the reference SPR spectrum, before immobilization of anti-BSA, was recorded with the PBS buffer. The PBS buffer was removed from the pool, and 0.25 $\mathrm{mg} / \mathrm{ml}$ anti-BSA solution was added and allowed to bind for 1 hour. After 1 hour the chip was washed with PBST for 8 minutes followed by the addition of PBS buffer. Then SPR spectrum was recorded once the response was stable. Next, the PBS buffer was removed and the casein blocking buffer was added for blocking the unbound sites. The blocking procedure was allowed for 30 minutes. The chip was then washed with PBST for 8 minutes followed by the addition of PBS buffer. Again the SPR spectrum was recorded after the response got stabilized. The PBS buffer was removed and $0.25 \mathrm{mg} / \mathrm{ml} \mathrm{BSA}$ solution was added to the pool. The binding was allowed for 30 minutes after which the SPR spectrum was recorded. The complete analysis was carried out at the incident wavelength of $670 \mathrm{~nm}$ with two prisms of refractive index values 1.597 and 1.456. All the SPR experiments were carried out at room temperature of $23 \pm 2{ }^{\circ} \mathrm{C}$.

\section{Results and discussion}

Figure 7 and 8 show the experiment results obtained for the prisms of refractive index values 1.597 and 1.456, respectively. In Fig. 7, curve 1 was recorded as the reference spectrum for the PBS buffer solution after the deposition of thiol and Protein A layers. Curve 2 was recorded in the PBS buffer solution after the immobilization of anti-BSA 
and the washing of unbound anti-BSA molecules with PBST. The shift between the SPR curves 1 and 2 was $0.101^{\circ}$ which confirmed a successful immobilization of anti-BSA on the sensor surface. Curve 3 was recorded after the binding of BSA. The small shift between curves 2 and 3 was $0.074^{\circ}$ due to the antibody-antigen binding. The same explanation holds for the three curves 1', 2' and 3' in Fig. 8. The shift between curves 1' and $2^{\prime}$ was $0.220^{\circ}$ and shift between curves $2^{\prime}$ and $3^{\prime}$ was $0.111^{\circ}$. Table 1 lists the data. The SPR spectrum recorded after casein blocking was not shown, as it overlapped the curve 2. As expected, the blocking of unbound sites did not result in any resonance shift. The resonance positions of spectra were obtained by fitting a polynomial of order 6 .

Comparing Fig. 7 and Fig. 8, the resonance position of spectra moved towards a higher angle region for the lower refractive index prism. Also, the shift between curves 1' and 2' was 2.18 times of that between curves 1 and 2, and the shift between curves $2^{\prime}$ and $3^{\prime}$ was 1.50 times of that between curves 2 and 3. Thus the SPR angular shift was larger for the smaller refractive index prism. This favoured the possibility of detection of much lower concentrations of biomaterials.

A drawback of using a low refractive index prism was the increase in the full width at half maximum (FWHM) of the SPR spectrum. With a high FWHM the accuracy of finding the resonance angle reduces. But the experimental spectra in Fig. 8 for the low refractive index prism showed that the increase in FWHM was not very large and the depth of the spectra was enough to find the resonance position with appreciable accuracy. Another point of concern was of working in a high incident angle region that causes the problem of grazing incidence. Tuning the resonance region near or above $80^{\circ}$ diminishes 
the precision of broad light beam hitting the prism-metal interface. Thus it must be avoided.

\section{Conclusion}

This report proposed the use of lower refractive index prism in SPR biosensing to improve the angular sensitivity of the sensor. According to the previous literature work, research groups mostly utilized a high refractive index prism and/or worked in a smaller angle region. It was shown through E3L model that the refractive index of dielectric layer contributing to the SPR is the effective refractive index between the bio-layers and the buffer surrounding. This permitted the use of a lower refractive index glass prism in biosensing. Thus, it is advisable to use a low refractive index prism and tune the resonance position to a relatively higher and measurable angle region near $70^{\circ}$ and increase the angular sensitivity. As a result the limit of detection will improve and the sensor will be capable of detecting further smaller concentrations of biochemicals.

\section{Acknowledgement}

The authors are thankful to Mr.Abhishek Chandra Srivastava, Hamamatsu University School of Medicine, Shizuoka, for valuable discussions and help in the experimental work. This work was partially supported by research grants of International Human Frontier Science Program Organization and $21^{\text {st }}$ Century COE program of Shizuoka University. 


\section{Biographies}

Gaurav Gupta received his B.Sc. degree in physics in 2002 from Delhi University, Delhi and M.Sc. degree in physics in 2004 from Indian Institute of Technology Delhi, New Delhi (India). He is currently is a doctor course student at the Graduate School of Electronic Science and Technology, Shizuoka University, Shizuoka (Japan).

Jun Kondoh was born in Shiga, Japan in 1967. He received his B.E., M.S., and Dr. Eng. degrees in opto-electric and mechanical engineering from Shizuoka University in 1990, 1992, and 1995, respectively. From April 1993 to March 1997, he was a research fellow of the Japan Society for the Promotion of Science. In 1996, he was a guest scientist at Karlsruhe Research Center, Germany. In April 1997, he joined the Department of Systems Engineering, Shizuoka University as a research associate and was promoted to an associate professor in 2003. Since 2001, he is visiting scientist of National Institute of Advanced Industrial Science and Technology (AIST). His current research interest is surface-wave-based sensors and actuators, such as SAW and surface plasmons. He is a member of the Acoustic Society of Japan, Japan Society of Applied Physics, the Institute of Electrical Engineers of Japan, the Institute of Electronics, Information and Communication Engineers, and the Electrochemical Society of Japan. 


\section{References}

[1] L.G. Fägerstam, Å. Frostell-Karlsson, R. Karlsson, R. Persson, I. Rönnberg, Biospecific interaction analysis using surface plasmon resonance detection applied to kinetic, binding site and concentration analysis, J. Chromatogr. 597 (1992) 397-410.

[2] Z. Salamon, H.A. Macleod, T. Gordon, Surface plasmon resonance spectroscopy as a tool for investigating the biochemical and biophysical properties of membrane protein systems. II: Applications to biological systems, Biochim. Biophys. Acta. 1331 (1997) 131-152.

[3] M. Beseničar, P. Maček, J. H. Lakey, G. Anderluh, Surface plasmon resonance in protein-membrane interactions, Chem. Phy. of Lipids 141 (2006) 169-178.

[4] J. Homola, I. Koudela, S.S. Yee, Surface plasmon resonance sensors based on diffraction gratings and prism couples: sensitivity comparison, Sens. Actuators, B, Chem 54 (1999) 16-24.

[5] G. Gupta, J. Kondoh, Tuning and sensitivity enhancement of surface plasmon resonance sensor, Sens. Actuator, B, Chem 122 (2007) 381-388.

[6] A. H. Schmid, S.E. Stanca, M.S. Thakur, K. R. Thampi, C. R. Suri, Site-directed antibody immobilization on gold substrate for surface plasmon resonance sensor, Sens. Actuators, B, Chem 113 (2006) 297-303.

[7] B.K. Oh, B.S. Chun, K.W. Park, W. Lee, W.H. Lee, J.W. Choi, Fabrication of protein G LB film for immunoglobulin G immobilization, Mater. Sci. Eng., C 24 (2004) 6569. 
[8] W. Lee, B.K. Oh, Y.M. Bae, S.H. Paek, W.H. Lee, J.W. Choi, Fabrication of selfassembled protein A monolayer and its application as an immunosensor, Biosens. Bioelectron. 19 (2003) 185-192.

[9] Y.M. Bae, B.K. Oh, W. Lee, W.H. Lee, J.W. Choi, Study on orientation of immunoglobulin G on protein G layer, Biosens. Bioelectron. 21 (2005) 103-110.

[10] H. Raether, Surface Plasmon on Smooth and Rough Surfaces and on Gratings, Vol. 111, Springer-Verlag, Berlin Heidelberg, 1988.

[11] M.A. Ordal, L.L. Long, R.J. Bell, S.E. Bell, R.R. Bell, R.W. Alexander Jr., C.A. Ward, Optical properties of the metals $\mathrm{Al}, \mathrm{Co}, \mathrm{Cu}, \mathrm{Au}, \mathrm{Fe}, \mathrm{Pb}, \mathrm{Ni}, \mathrm{Pd}, \mathrm{Pt}, \mathrm{Ag}, \mathrm{Ti}$, and W in the infrared and far infrared, Appl. Opt. 22 (1993) 1099-1119.

[12] P. Lorrain, D. Corson, Electromagnetic Fields and Waves, 2nd ed., CBS Publishers and Distributors, New Delhi, 2001.

[13] W.N. Hansen, Electric Field Produced by the Propagation f Plane Coherent Electromagnetic Radiation in a Stratified Medium, J. Opt. Soc. Am. 58 (1968) 380390. 


\section{Figure captions}

Fig. 1. (a) Multiple layer SPR biosensor configuration; (b) Effective 3-layer SPR.

Fig. 2. (a) 4-layer configuration; (b) E3L configuration. The value of $\varepsilon_{\mathrm{m}}=-14.77+\mathrm{i} 1.18$; $n_{\text {eff }}=$ ?.

Fig. 3. Numerically generated SPR spectra for 4-layer and 3-layer models of Fig. 2.

Fig. 4. Plot of effective refractive index $\left(n_{\text {eff }}\right)$ as a function of thickness of bio-layer $\left(d_{\text {bio }}\right)$. The value of $n_{\mathrm{p}}=1.70, \varepsilon_{\mathrm{m}}=-14.77+\mathrm{i} 1.18, d_{\mathrm{m}}=50 \mathrm{~nm}$ and $\lambda=670 \mathrm{~nm}$.

Fig. 5. Time-averaged square field distribution across the media. (a) 4-layer configuration; (b) Effective 3-layer configuration.

Fig. 6. Schematics of prism-type SPR sensor setup working in angular interrogation mode. LD: laser diode; BS: beam splitter; P: polarizer; PD1: photodiode1; PD2: photodiode2; ADC: analog-to-digital converter.

Fig. 7. SPR spectra for immunosensor experiment with prism of refractive index 1.597. Curve 1 is the reference spectrum in PBS buffer. Curve 2 is recorded in PBS buffer after anti-BSA immobilization. Curve 3 is recorded in PBS buffer after BSA binding.

Fig. 8. SPR spectra for immunosensor experiment with prism of refractive index 1.456 . Curve 1' is the reference spectrum in PBS buffer. Curve 2' is recorded in PBS buffer after anti-BSA immobilization. Curve $3^{\prime}$ is recorded in PBS buffer after BSA binding. 


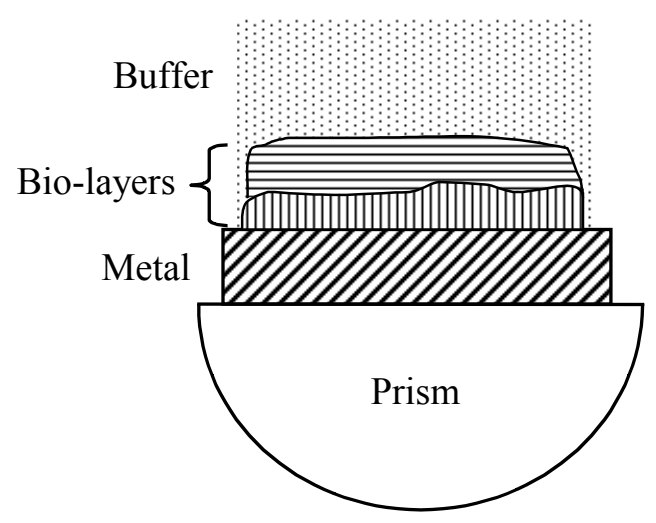

(a)

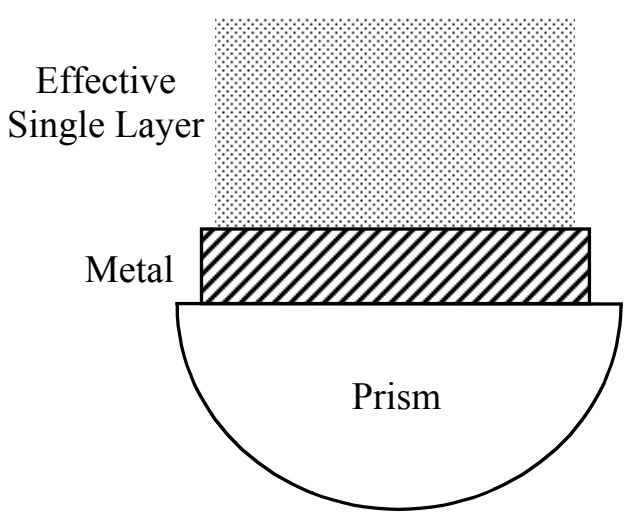

(b)

Fig. 1. (a) Multiple layer SPR biosensor configuration; (b) Effective 3-layer SPR configuration.

Gaurav Gupta et al.

Figure Size : 1/1 


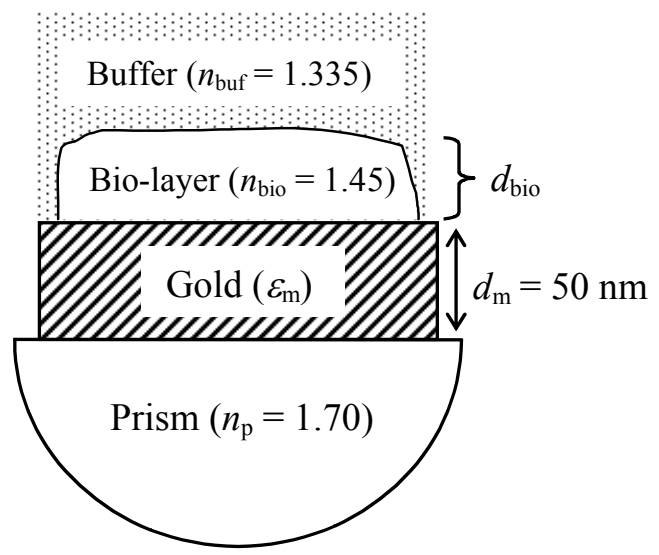

(a)

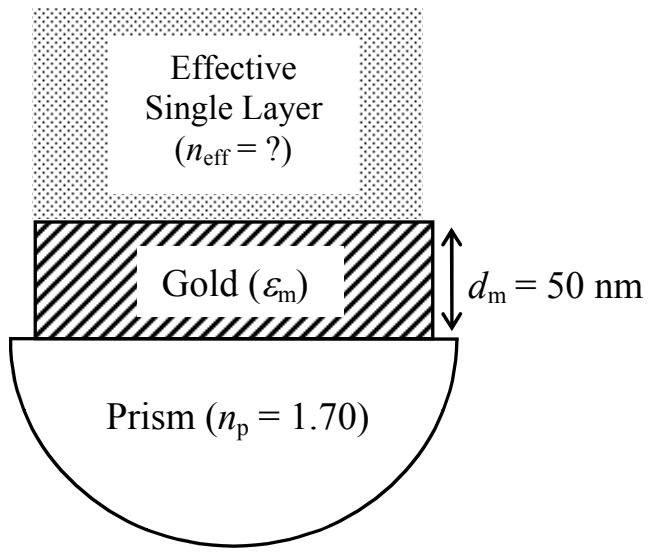

(b)

Fig. 2. (a) 4-layer configuration; (b) E3L configuration. The value of $\varepsilon_{\mathrm{m}}=-14.77+\mathrm{i} 1.18 ; n_{\mathrm{eff}}=$ ?

\section{Gaurav Gupta et al.}

Figure Size : 1/1 


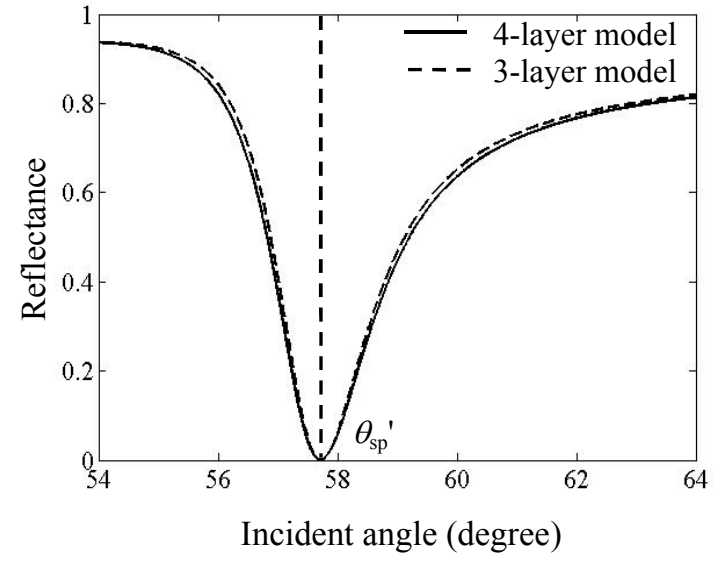

Fig. 3. Numerically generated SPR spectra for 4-layer and 3-layer models of Fig. 2.

Gaurav Gupta et al.

Figure Size : 1/1 


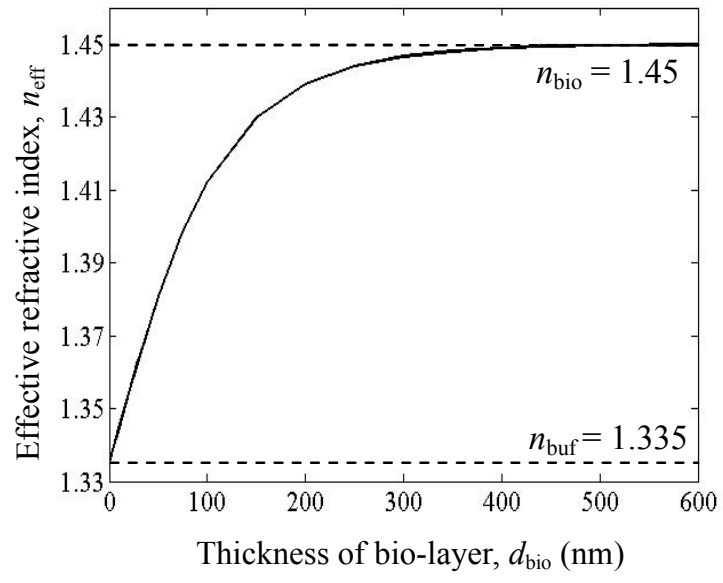

Fig. 4. Plot of effective refractive index ( $\left.n_{\text {eff }}\right)$ as a function of thickness of bio-layer $\left(d_{\text {bio }}\right)$. The value of $n_{\mathrm{p}}=1.70, \varepsilon_{\mathrm{m}}=-14.77+\mathrm{i} 1.18, d_{\mathrm{m}}=50 \mathrm{~nm}$ and $\lambda=670 \mathrm{~nm}$.

\section{Gaurav Gupta et al.}

Figure Size : 1/1 


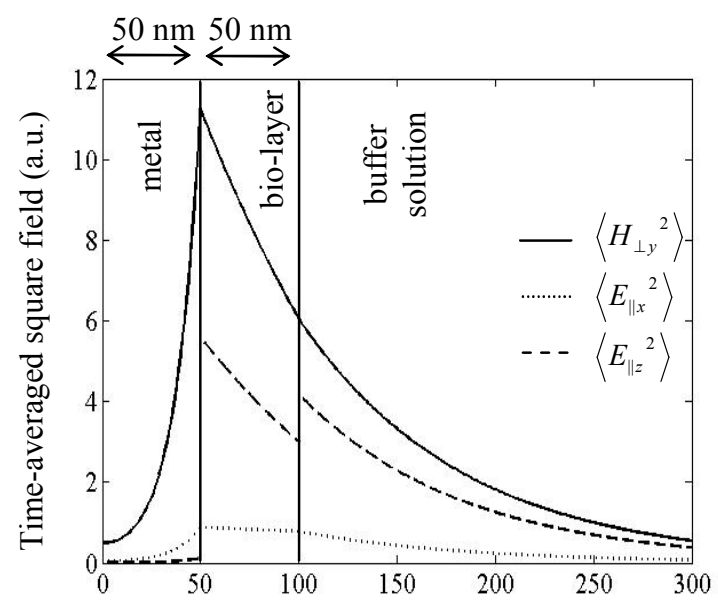

Distance across the metal-biolayer-buffer (nm)

(a) $\theta_{\mathrm{sp}}=60.56^{\circ}$

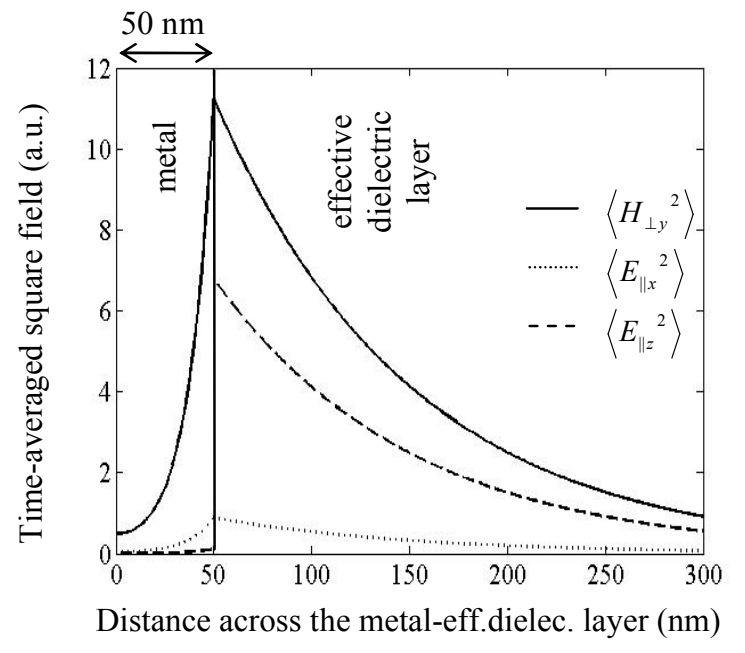

(b) $\theta_{\mathrm{sp}}=60.56^{\circ}$

Fig. 5. Time-averaged square field distribution across the media. (a) 4-layer configuration; (b) Effective 3-layer configuration.

\section{Gaurav Gupta et al.}

Figure Size : 1/1 


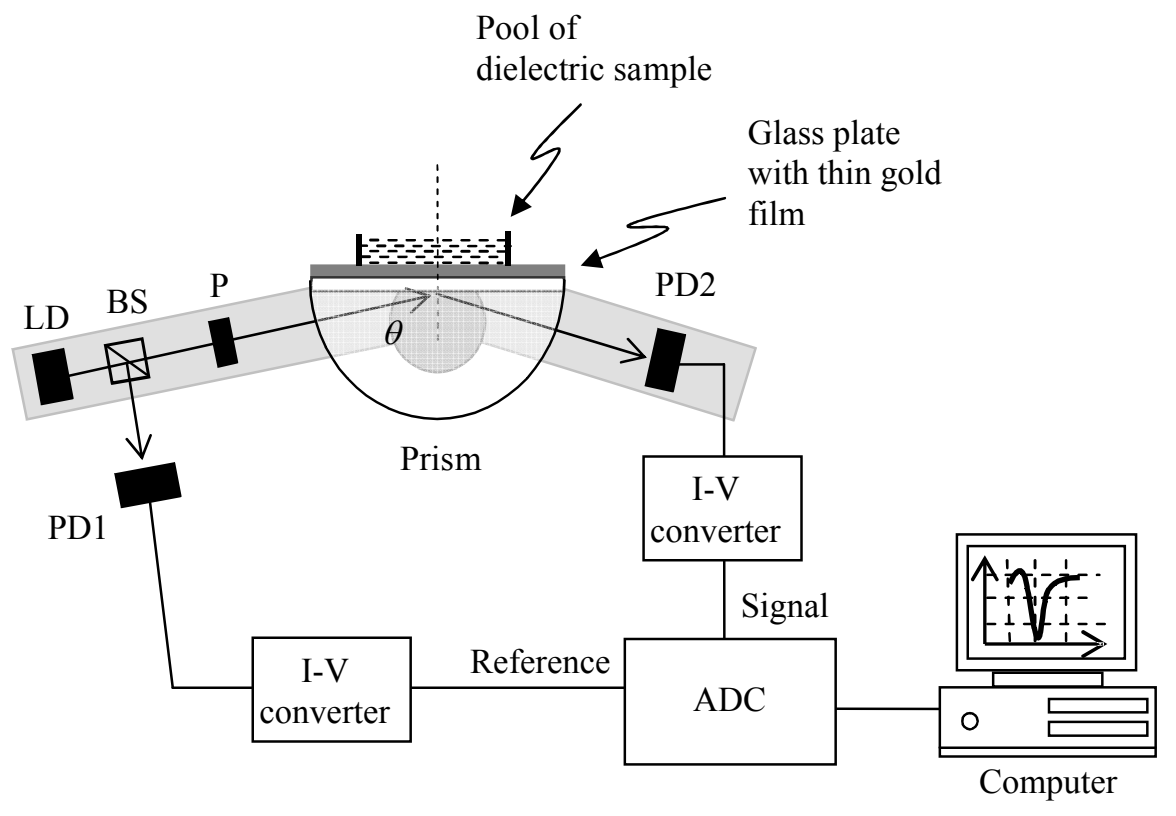

Fig. 6. Schematics of prism-type SPR sensor setup working in angular interrogation mode. LD: laser diode; BS: beam splitter; P: polarizer; PD1: photodiode1; PD2: photodiode2; ADC: analog-to-digital converter.

\section{Gaurav Gupta et al.}

Figure Size : 1/1 


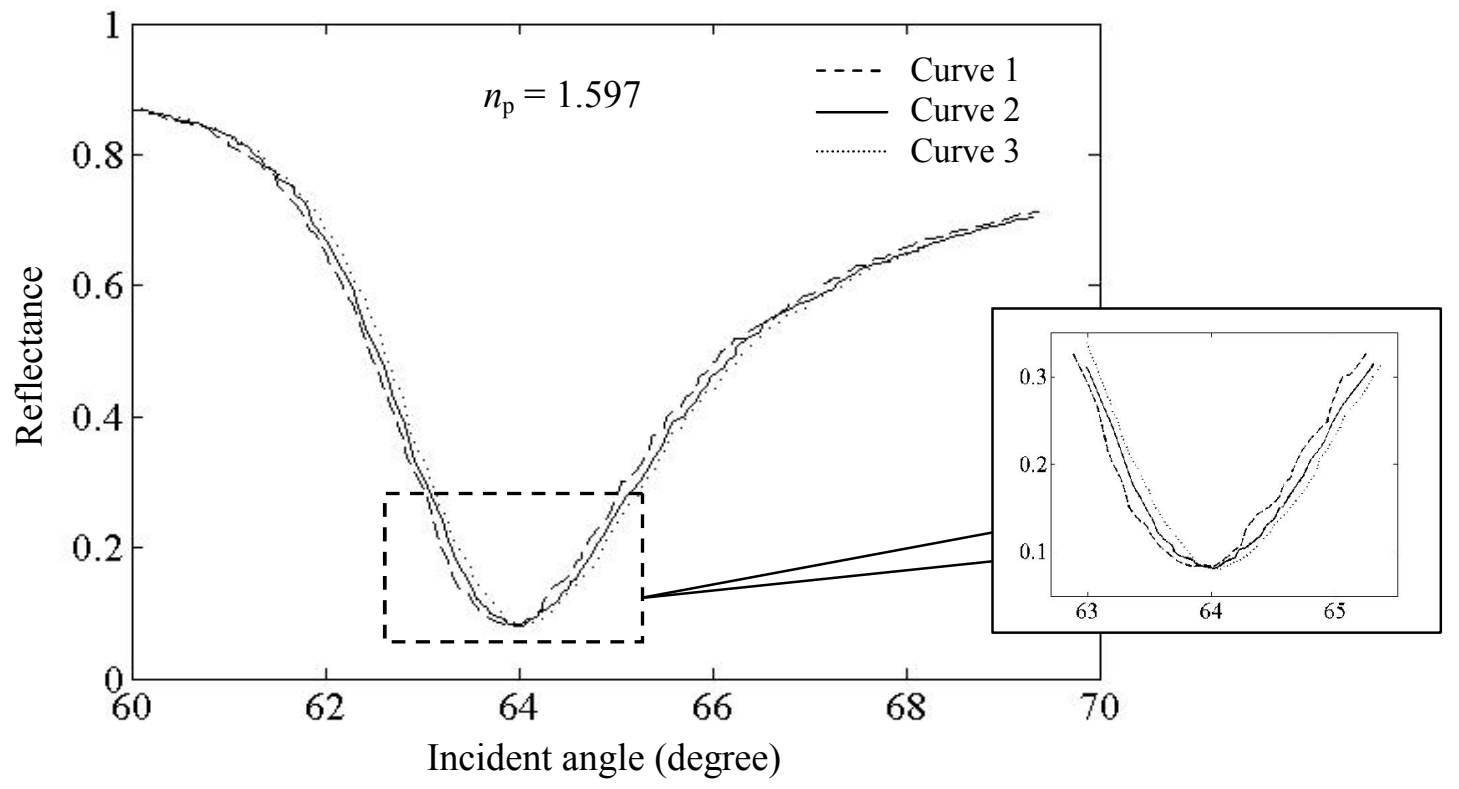

Fig. 7. SPR spectra for immunosensor experiment with prism of refractive index 1.597. Curve 1 is the reference spectrum in PBS buffer. Curve 2 is recorded in PBS buffer after anti-BSA immobilization. Curve 3 is recorded in PBS buffer after BSA binding.

Gaurav Gupta et al.

Figure Size : 1/1 


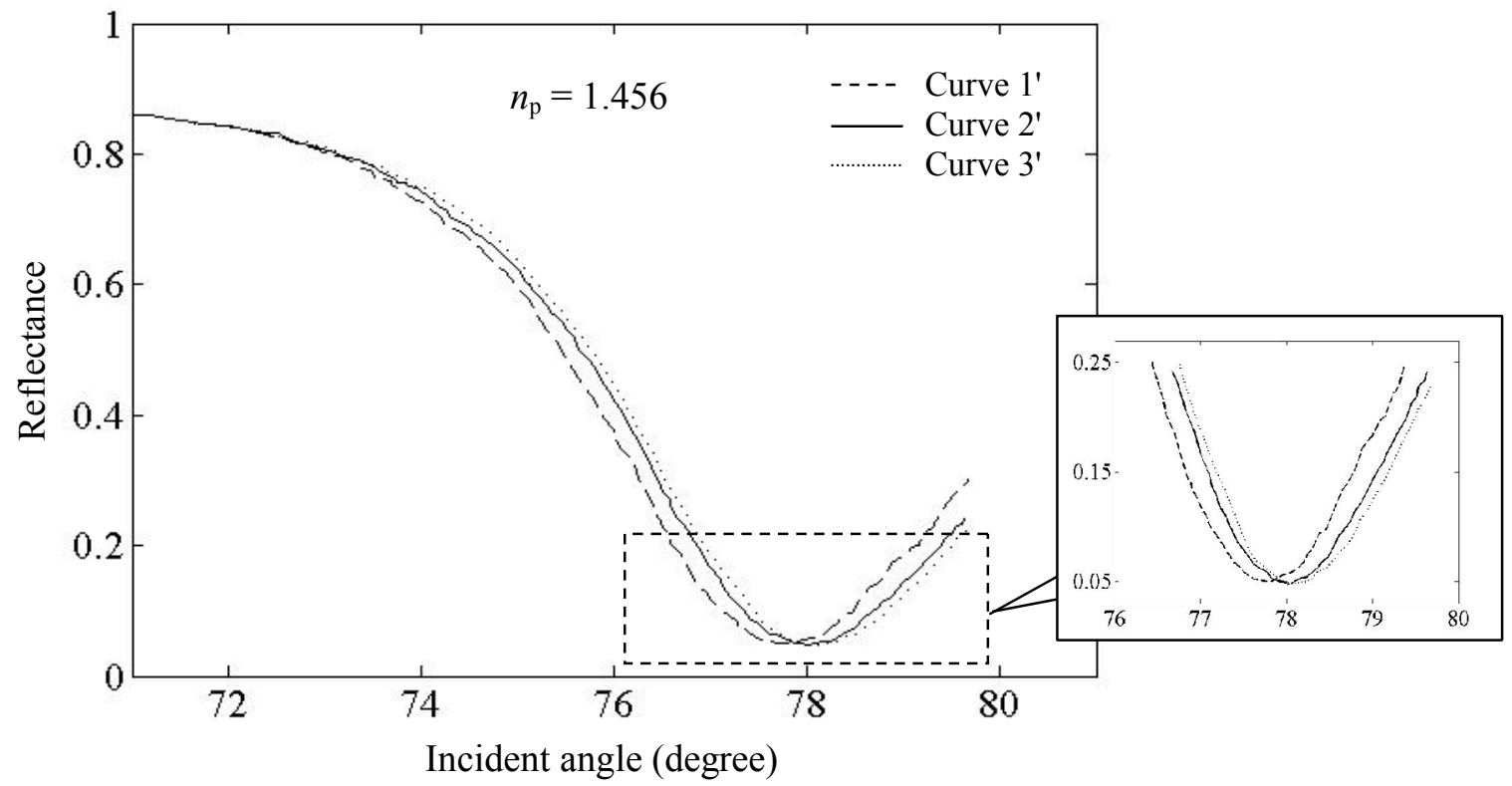

Fig. 8. SPR spectra for immunosensor experiment with prism of refractive index 1.456. Curve 1' is the reference spectrum in PBS buffer. Curve 2' is recorded in PBS buffer after anti-BSA immobilization. Curve 3 ' is recorded in PBS buffer after BSA binding.

Gaurav Gupta et al.

Figure Size : 1/1 
Table 1 Experimental data from SPR immunosensor experiments with two prism materials.

\begin{tabular}{|c|c|c|}
\hline$n_{\mathrm{p}}$ & $\begin{array}{c}\text { Resonance shift after } \\
\text { anti-BSA immobilization } \\
\text { (degree) }\end{array}$ & $\begin{array}{c}\text { Resonance shift after } \\
\text { BSA binding } \\
\text { (degree) }\end{array}$ \\
\hline 1.597 & 0.101 & 0.074 \\
\hline 1.456 & 0.220 & 0.111 \\
\hline
\end{tabular}

Gaurav Gupta et al.

Figure Size : 1/1 